\title{
Pointwise Convergent Nets of Holomorphic Automorphisms of the Unit Ball of Cartan Factors
}

\author{
J.M. ISIDRO* and L. STACHÓ
}

\begin{abstract}
A classical result due to $H$. Cartan states that if a sequence $\left(h_{n}\right)$ of holomorphic authomorphisms of the unit disk $\Delta$ of $\mathbb{C}$ is pointwise convergent on $\Delta$ to a limit $h$ and $\left(h_{n}(0)\right)$ is bounded away from the boundary $\partial(\Delta)$, then $h$ is a holomorphic automorphism of $\Delta$. The analogous result for the open unit ball $D$ of a complex Banach space $E$ is not true in general. Here we consider pointwise convergent nets $\left(h_{i}\right)$ of holomorphic automorphisms of the unit balls of those Banach spaces known as special Cartan factors and establish a sufficient condition for the pointwise limit $h=\lim _{i} h_{i}$ to be a holomorphic automorphism of $D$.
\end{abstract}

\footnotetext{
* Supported by Xunta de Galicia, project Xuga 2070290 1991 Mathematics Subject Classification: 46G20, 32M15 Servicio publicaciones Univ. Complutense. Madrid, 1995.
} 


\section{INTRODUCTION}

Consider the open unit disk $\Delta$ of the complex plane $\mathbb{C}$ and denote by $G$ the group of all holomorphic automorphisms of $\Delta$. Each $h$ in $G$ admits a decomposition $h=g_{a} \circ u_{h}$ where $u_{h}$ is a linear isometry of $\mathbb{C}, a=h(0)$ and $g_{a} \in G$ satisfies $g_{a}(0)=a$. It is a classical result that if a sequence $\left(h_{n}: n \in \mathbb{N}\right)$ in $G$ is pointwise convergent on $\Delta$ to a limit $h: \Delta \rightarrow \mathbb{C}$ and $\left(h_{n}(0): n \in \mathbb{N}\right)$ is bounded away from the boundary TI of $\Delta$, then $h$ belongs to $G$ and $h_{n}$ tends to $h$ uniformly on compact subsets of $\Delta$. We remark that the restriction on $\left(h_{n}(0): n \in \mathbb{N}\right)$ can not be dropped; however, there is no restriction on the linear components $u_{n}$ of the $h_{n}, n \in \mathbb{N}$, i.e., once the condition $\lim _{n} h_{n}(0) \notin \mathbb{T}$ is fulfilled we have $h \in G$ and $h_{n}$ tends to $h$ in the topology of $G$.

A natural generalization of $\Delta$ to higher (and even infinite) dimensions are the bounded symmetric domains in their standard realizations. These are precisely the open unit balls $D$ of those complex Banach spaces $E$ for which the group $G$ of all holomorphic automorphisms acts transitively on $D$. It is therefore reasonable to ask whether the above stated result still holds in the new situation.

Even in infinite dimensions, $G$ is a topological group when endowed with the topology of (local) uniform convergence on $D$, and every holomorphic automorphism $h \in G$ admits a canonical decomposition $h=g_{h(0)} \circ u_{h}$ where $g_{h(0)} \in G, g_{z}(0)=z(z \in D)$, the mapping $D \rightarrow G$ given by $z \mapsto g_{z}$ is continuous and $u_{h}$ is suitable linear $E$-unitary operator. We shall see (Section 4 ) that the non-linear part $g_{h(0)}$ can easily be ruled out.

On the other hand, in infinite dimensions even the simplest case provides new features. Consider a Hilbert space $H$ (a factor of type 1) with an orthonormal basis $\left\{e_{1}, e_{2}, \ldots\right\}$. The sequence $U_{1}, U_{2}, \ldots$ of reflections in $H$ acting on the basis as $U_{n}\left(e_{k}\right):=(-1)^{1+\delta_{n k}} e_{k}(n, k=$ $1,2, \ldots$ ) converges pointwise but not (locally) uniformly on $H$. Moreover there are pointwise convergent sequences of $H$-unitary operators whose inverses diverge. A typical example is given by $V_{n}\left(e_{k}\right):=e_{\tau_{n}(k)}(n, k=$ $1,2, \ldots)$ where $\tau_{n}$ denotes the cyclic permutation of the first $n$ indices (i.e. $\tau(k):=k+1$ for $k\langle n, \tau(n):=1$ and $\tau(k):=k$ if $k>n$ ). The pointwise limit of the $V_{n}$ is a unilateral shift of $H$, which is not an $H$-unitary operator. 
Our considerations in this work are inspired by these two examples. Infinite dimensional Cartan factors can be represented as spaces of linear operators acting between Hilbert spaces. Their atoms are operators of rank at most two in these representations, and we may control the effect of pointwise convergent nets of linear automorphisms by investigating some attached nets of Hilbert space unitary operators. Hence we achieve relevant information concerning pointwise convergent nets of holomorphic automorphism in Cartan factors and in spaces of Cartan factor-valued continuous functions. In particular, we establish a sufficient condition for the pointwise limit $h=\lim _{\alpha} h_{\alpha}$ of a net in $G$ to be a holomorphic automorphism of $D$.

Notation and background material Let $D$ be a bounded domain in a complex Banach space $E$. A function $f: D \rightarrow E$ is called holomorphic if for every $a \in D$ the Fréchet derivative $f^{\prime}(a) \in \mathcal{L}(E)$ exists. A holomorphic automorphism of $D$ is a bijection $h: D \rightarrow D$ such that $h$ and $h^{-1}$ are holomorphic, and $G:=\operatorname{Aut}(D)$ denotes the group of all holomorphic automorphisms of $D$ endowed with the topology of local uniform convergence. Then $D$ is called symmetric if to every $a \in D$ there is an $s=s_{a} \in G$ with $s^{2}=$ id having $a$ as isolated fixed point. It is known that $G$ acts transitively on $D$, and that $D$ is biholomorphically equivalent to the open unit ball of $E$ when renormed adequately (see [9] for details).

A complex Banach space $E$ is called a $J B^{*}$-triple if the open unit ball $D \subset E$ is symmetric, or equivalently if the automorphism group $G=$ Aut $(D)$ acts transitively on $D$. Then there exists a uniquely determined continuous ternary operation (called the Jordan triple product on $E$ ) $(x, y, z) \mapsto\{x y z\}$ from $E^{3}$ to $E$ such that, by writing $x$ ㅁ for the linear operator $z \mapsto\{x y z\}$ on $E$, the following axioms are satisfied

$\left(J_{1}\right)\{x y z\}$ is symmetric bilinear in the outer variables $x, z$ and conjugate linear in the inner variable $y$

$\left(J_{2}\right)[x \square x, y \square y]=\{x x y\} \square y+y \square\{y x x\}$

$\left(J_{3}\right) x \square x$ is hermitian and has spectrum $\geq 0$

$\left(J_{4}\right)\|\{x x x\}\|=\|x\|^{3}$

for all $x, y \in E$ and [,] being the commutator product of linear operators. 
On the other hand, every complex Banach space $E$ admitting a continuous mapping $\{,$,$\} with \left(J_{1}\right)-\left(J_{4}\right)$ is a $J B^{*}$-triple. The notion of triple automorphism can be introduced in the natural way, and the group $\operatorname{Aut}(E)$ of all triple automorphisms of $E$ coincides with the group of all surjective linear isometries of $E$. An element $e \in E$ is called a tripotent if $\{e e e\}=e$ and a tripotent $e$ is called an atom in $E$ if $\{e E e\}=\mathbb{C e}$. The set at $(E)$ of all atoms is closed in $E$ and, except for $e=0$, is contained in $\partial D$. The following examples of $J B^{*}$-triples are known as Cartan factors of type $k,(k=1,2,3,4)$ :

Type 1: Are the spaces $\mathcal{L}(H, K)$ of all bounded linear operators $x$ : $H \rightarrow K$ where $H$ and $K$ are complex Hilbert spaces.

Types 2,3: Let $H$ be a complex Hilbert space with a conjugation - and let ${ }^{t}$ be the induced transposition on $\mathcal{L}(H)$; for $\varepsilon=1$ and $\varepsilon=-1$ the spaces $\left\{x \in \mathcal{L}(H) \mid x^{t}=\varepsilon x\right\}$ are called Cartan factors of types 2 and 3 , respectively.

Type 4: Also called spin factors, are defined as any norm closed selfadjoint complex subspace $\mathcal{U} \subset \mathcal{L}(H)$ such that $\left\{x^{2} \mid x \in \mathcal{U}\right\} \subset$ $\mathbb{C} 1_{\mathrm{H}}$ and $\operatorname{dim} \cdot \mathcal{U}>2$.

In all these cases the triple product is defined by $\{x y z\}:=\left(x y^{*} z+\right.$ $\left.z y^{*} x\right) / 2$, where $y^{*}$ denotes the adjoint of the operator $y$. If $\mathcal{U}$ is a spin factor, then for every pair $a, b \in \mathcal{U}$ we have $a b^{*}+b^{*} a=\langle a \mid b\rangle 1_{H}$ for some $\langle a \mid b\rangle \in \mathbb{C}$, and $\langle. \mid$.$\rangle is an inner product in U$ whose norm $\||\cdot \||$ is equivalent to the operator norm $\|\cdot\|$. We refer to $\mathcal{H}:=(\mathcal{U},\|\cdot \cdot\| \|)$ as the Hilbert space associated to $\mathcal{U}$.

Besides these special Cartan factors, there are two exceptional Car$\tan$ factors which are finite dimensional spaces (see [11]). If $\Omega$ is a locally compact Hausdorff space and $E$ is a $J B^{*}$-triple, then $\mathcal{C}_{0}(\Omega, E)$, the space of continuous $E$-valued functions that vanish at infinity with the pointwise triple product and the norm of the supremum, is also a $J B^{*}$-triple.

For a special Cartan factor $E$, we shall need the characterization of its atoms that is given in [4] and the representation of its surjective linear isometries given in [10] for $k=1$, in [5] for $k=2,3$ and in [6] for $k=4$.

The group of surjective linear isometries (or unitary operators) on a Banach space $E$ will be denoted bv $\mathcal{U}(E)$. Whenever $\left(x_{*}, i \in I\right)$ is 
a net in $E$ and $\lim _{i} x_{i}=x$ holds for some $x \in E$ with respect to the norm topology, we shall say that $\left(x_{i}\right)$ converges in $E$ and write $x_{i} \rightarrow x$ or $x_{i}=x+o(1)$. We shall also write $x_{i} \rightarrow x$ and $x_{i}=x+o(1)$ if $\lim _{i} x_{i}=x$ holds with respect to some other topology $\tau$ on $E$, but then an explicit reference to $\tau$ will be made. In particular, the abbreviations SOT and SSOT stand respectively for the strong operator and the strong star operator topologies on $\mathcal{L}(H, K)$.

\section{SOME PRELIMINARY RESULTS}

1.1 Lemma. Let $H$ and $K$ be the Hilbert spaces and suppose that $\left(e_{i}\right)$ and $\left(f_{i}\right), i \in I_{f}$ are nets of unit vectors in $K$ and $H$, respectively, such that the operators $e_{i} \otimes f_{i}^{*}:=\left\langle\cdot, f_{i}\right\rangle e_{i}$ converge in the $S O T$ of $\mathcal{L}(H, K)$. Then $\left(f_{i}\right)$ converges in $H$ if and only if $\left(e_{i}\right)$ converges in $K$.

Proof. If $\left(f_{i}\right)$ converges in $H$ then we have

$$
\begin{aligned}
\left\|e_{i}-e_{j}\right\| & =\left\|\left(e_{i}-e_{j}\right) \otimes f_{i}^{*}\right\|=\left\|e_{i} \otimes f_{i}^{*}-e_{j} \otimes f_{j}^{*}+e_{j} \otimes\left(f_{j}-f_{i}\right)^{*}\right\| \\
& \leq\left\|e_{i} \otimes f_{i}^{*}-e_{j} \otimes f_{j}^{*}\right\|+\left\|f_{j}-f_{i}\right\| \rightarrow 0 .
\end{aligned}
$$

Thus $\left(e_{i}\right)$ is a Cauchy net. The converse argument is similar.

1.2 Proposition. Let $H$ and $K$ be Hilbert spaces and let $\left(e_{i}\right)$ and $\left(f_{i}\right), i \in I$, be nets of unit vectors in $K$ and $H$, respectively, such that the operators $\left(e_{i} \otimes f_{i}^{*}\right)$ converge in $\mathcal{L}(H, K)$. Then there is a net $\left(\alpha_{i}: i \in I\right)$ in 'II' such that $\left(\alpha_{i} e_{i}\right)$ and $\left(\alpha_{i} f_{i}\right)$ converge in $K$ and $H$ respectively.

Proof. Since at $[\mathcal{L}(H, K)]$ is closed we can find unit vectors $e \in K$ and $f \in H$ such that

$$
e_{i} \otimes f_{i}^{*} \rightarrow e \otimes f^{*}
$$

holds in $\mathcal{L}(H, K)$. By writting $\langle\cdot, \cdot\rangle$ for the scalar product both in $H$ and $K$ we have

$$
\begin{aligned}
\left\langle e_{i}, e\right\rangle\left\langle f, f_{i}\right\rangle= & \left\langle\left(e_{i} \otimes f_{i}^{*}\right) f, e\right\rangle \rightarrow \\
& \left\langle\left(e \otimes f^{*}\right) f, e\right\rangle=\langle e, e\rangle\langle f, f\rangle=1
\end{aligned}
$$


Therefore the definition $\alpha_{i}:=\left\langle e, e_{i}\right\rangle /\left|\left\langle e, e_{i}\right\rangle\right|$ makes sense for $i \in I, i \geq$ $i_{0}$, and

$$
\begin{aligned}
\left\|\alpha_{i} e_{i}-e\right\|^{2} & =\left\|\alpha_{i} e_{i}\right\|^{2}+\|e\|^{2}-2 \mathcal{R} e\left\langle\alpha_{i} e_{i}, e\right\rangle \\
& =2\left(1-\mathcal{R e} \frac{\left\langle e, e_{i}\right\rangle\left\langle e_{i}, e\right\rangle}{\mid\left\langle e, e_{i}\right\rangle}\right)=2\left(1-\left|\left\langle e, e_{i}\right\rangle\right|\right) \rightarrow 0 .
\end{aligned}
$$

Thus $\left(\alpha_{i} e_{i}\right)$ converges in $K$. Since $e_{i} \otimes f_{i}^{*}=\left(\alpha_{i} e_{i}\right) \otimes\left(\alpha_{i} f_{i}\right)^{*}$ for $i \in I$, by $(1.1)$ the net $\left(\alpha_{i} f_{i}\right)$ converges in $H$.

1.3 Corollary. Let $H$ be a Hilbert space and - a conjugation on $H$. If $\left(e_{i}: i \in I\right)$ is a net of unit vectors in $H$ such that the operators $e_{i} \otimes \bar{e}_{i}^{*}$ converge in $\mathcal{L}(H)$, then there is a net $\left(\varepsilon_{i}: i \in I\right)$ in $\{-1,1\}$ stuch that $\left(\varepsilon_{i} e_{i}\right)$ converges in $H$.

Proof. By (1.2) we can find a net $\left(\alpha_{i}\right)$ in $\mathbb{T}$ such that $\left(\alpha_{i} e_{i}\right)$ and $\left(\alpha_{i} \vec{e}_{i}\right)$ converge in $H$. By conjugation also $\left(\overline{\alpha_{i} e_{i}}\right)$ converges in $H$, and so $e_{i} \otimes \bar{e}_{i}^{*}$ and $\alpha_{i} e_{i} \otimes \overline{\alpha_{i} e_{i}^{*}}=\alpha_{i}^{2} e_{i} \otimes \bar{e}_{i}^{*}$ converge in $\mathcal{L}(H)$. Therefore $\alpha_{i}^{2} \rightarrow \alpha$ for some $\alpha \in \mathbb{I}$. Fix a determination of $\sqrt{\alpha}$. Then $\varepsilon_{i}:=$ $\operatorname{sgn}\left(\operatorname{Re}\left(\alpha_{i} / \sqrt{\alpha}\right)\right)$, which is well-defined for $i \geq i_{0}$, suits our requirements.

1.4 Proposition. Let $H$ be a Hilbert space and " a conjugation on $H$. Let $\left(e_{i}\right)$ and $\left(f_{i}\right)$ be nets of unit vectors in $H$ such that $e_{i} \perp f_{i}, i \in I$, and the operators $e_{i} \otimes \bar{f}_{i}^{*}-f_{i} \otimes \bar{e}_{i}^{*}$ converge in $\mathcal{L}(H)$. Then there are nets $\left(\alpha_{i}\right)$ and $\left(\beta_{i}\right)$ in $\mathbb{C}$ such that $\left|\alpha_{i}\right|^{2}+\left|\beta_{i}\right|^{2}=1, i \in I$, and both $\alpha_{i} e_{i}+\beta_{i} f_{i}$ and $-\bar{\beta}_{i} e_{i}+\bar{\alpha}_{i} f_{i}$ converge in $H$.

Proof. Since at $[\mathcal{L}(H)]$ is closed in $\mathcal{L}(H)$, there exists an orthonormal frame $\{e, f\}$ in $H$ with

$$
e_{i} \otimes \ddot{f}_{i}^{*}-f_{i} \otimes \bar{e}_{i}^{*} \rightarrow e \otimes \bar{f}^{*}-f \otimes \bar{e}^{*} \quad \text { in } \mathcal{L}(H)
$$

Set $\lambda_{i}:=\left\langle e, e_{i}\right\rangle, \mu_{i}:=\left\langle e, f_{i}\right\rangle$ and $g_{i}:=\lambda_{i} e_{i}+\mu_{i} f_{i}, h_{i}:=-\overline{\mu_{i}} e_{i}+\overline{\lambda_{i}} f_{i}$ for all $i \in I$. Then

$$
g_{i} \otimes \bar{h}_{i}^{*}-h_{i} \otimes \bar{g}_{i}^{*}=\left(\left|\lambda_{i}\right|^{2}+\left|\mu_{i}\right|^{2}\right)\left(e_{i} \otimes \bar{f}_{i}^{*}-f_{i} \otimes \bar{e}_{i}^{*}\right)
$$


By the reflexivity of $H$ we can choose a subnet $\left(i_{k}: k \in \mathcal{K}\right)$ and two vectors $e_{0}, f_{0} \in H$ such that

$$
\begin{aligned}
& e_{i_{k}} \rightarrow e_{0}, \quad f_{i_{k}} \rightarrow f_{0} \quad \text { (weak convergence in } H \text { ) } \\
& \lambda_{i_{k}} \rightarrow \lambda, \quad \mu_{i_{k}} \rightarrow \mu \quad \text { where } \lambda:=\left\langle e, e_{0}\right\rangle, \mu:=\left\langle e, f_{0}\right\rangle .
\end{aligned}
$$

Since for all $x, y \in H$ we have

$$
\begin{aligned}
\left\langle\left(e_{i k} \otimes \overline{f_{i k}^{*}}\right) x, y\right\rangle & =\left\langle e_{i k}, y\right\rangle\left\langle x, f_{i k}\right\rangle \rightarrow \\
& \left(e_{0}, y\right\rangle\left\langle x, f_{0}\right\rangle=\left\langle\left(e_{0} \otimes \overline{f_{0}^{*}}\right) x, y\right\rangle,
\end{aligned}
$$

it follows that

$$
\begin{aligned}
& e_{0} \otimes \bar{f}_{0}^{*}-f_{0} \otimes \bar{e}_{0}^{*}=e \otimes \bar{f}^{*}-f \otimes \bar{e}^{*} \\
& g_{0} \otimes \bar{h}_{0}^{*}-h_{0} \otimes \bar{g}_{0}^{*}=\left(|\lambda|^{2}+|\mu|^{2}\right)\left(e \otimes \bar{f}^{*}-f \otimes \bar{e}^{*}\right)
\end{aligned}
$$

where

$$
g_{0}:=\lambda e_{0}+\mu f_{0}, \quad h_{0}:=-\bar{\mu} e_{0}+\bar{\lambda} f_{0} .
$$

A comparison of the ranges in (1) gives the existence of $\alpha, \beta, \gamma, \delta \in \mathbb{C}$ with

$$
e_{0}=\alpha e+\beta f, \quad f_{0}=\gamma e+\delta f .
$$

\section{Hence}

$$
e \otimes \bar{f}^{*}-f \otimes \bar{e}^{*}=e_{0} \otimes \bar{f}_{0}^{*}-f_{0} \otimes \bar{e}_{0}^{*}=(\alpha \delta-\beta \gamma)\left(e \otimes \bar{f}^{*}-f \otimes \bar{e}^{*}\right)
$$

Thus

$$
\alpha \delta-\beta \gamma=1 \text { and }\left\|e_{0}\right\|^{2}=|\alpha|^{2}+|\beta|^{2} \leq 1,\left\|f_{0}\right\|^{2}=|\gamma|^{2}+|\delta|^{2} \leq 1
$$


By the Schwarz inequality, the relation $1=\alpha \delta-\beta \gamma=\langle(\alpha, \beta),(\bar{\delta},-\bar{\gamma})\rangle$ may hold only if $\alpha=\bar{\delta}$ and $\beta=-\bar{\gamma}$. Therefore

$$
\begin{aligned}
& e_{0}=\alpha e+\beta f \quad f_{0}=-\vec{\beta} e+\bar{\alpha} f \\
& \alpha=\left\langle e_{0}, e\right\rangle=\bar{\lambda},-\bar{\beta}=\left\langle f_{0}, e\right\rangle=\bar{\mu}, \gamma=-\bar{\beta}=\bar{\mu}, \delta=\bar{\alpha}=\lambda \\
& 1=\alpha \delta-\beta \gamma=|\lambda|^{2}+|\mu|^{2} \\
& g_{0}=\lambda e_{0}+\mu f_{0}=\left(|\lambda|^{2}+|\mu|^{2}\right) e=e, h_{0}=\left(|\lambda|^{2}+|\mu|^{2}\right) f=f .
\end{aligned}
$$

From the above considerations we can conclude that

$$
\begin{aligned}
& \lambda_{i_{k}} e_{i_{k}}+\mu_{i_{k}} f_{i_{k}} \rightarrow e,-\bar{\mu}_{i_{k}} e_{i_{k}}+\bar{\lambda}_{i_{k}} f_{i_{k}} \rightarrow f(\text { weak convergence in } H) \\
& \left|\left\langle e, e_{i_{k}}\right\rangle\right|^{2}+\left|\left\langle e, f_{i_{k}}\right\rangle\right|^{2}=\left|\lambda_{i_{k}}\right|^{2}+\left|\mu_{i_{k}}\right|^{2} \rightarrow 1 .
\end{aligned}
$$

Notice that these relations are valid for arbitrary weakly convergent subnets $\left(e_{i k}\right),\left(f_{i k}\right)$. Since the closed unit ball of $H$ is weakly compact, the same statement holds for the whole nets, i.e.

$$
\begin{aligned}
& \lambda_{i} e_{i}+\mu_{i} f_{i} \rightarrow e,-\bar{\mu}_{i} e_{i}+\bar{\lambda}_{i} f_{i} \rightarrow f(\text { weak convergence in } H) \\
& \left|\left\langle e, e_{i}\right\rangle\right|^{2}+\left|\left\langle e, f_{i}\right\rangle\right|^{2} \rightarrow 1
\end{aligned}
$$

A weakly convergent net of unit vectors whose limit is a unit vector converges in norm, hence

$$
\begin{aligned}
& \alpha_{i}:=\frac{\lambda_{i}}{\sqrt{\left|\lambda_{i}\right|^{2}+\left|\mu_{i}\right|^{2}}}=\frac{\left\langle e, e_{i}\right\rangle}{\sqrt{\left|\left\langle e, e_{i}\right\rangle\right|^{2}+\left|\left\langle e, f_{i}\right\rangle\right|^{2}}} \\
& \beta_{i}:=\frac{\mu_{i}}{\sqrt{\left|\lambda_{i}\right|^{2}+\left|\mu_{i}\right|^{2}}}=\frac{\left\langle e, f_{i}\right\rangle}{\sqrt{\left|\left\langle e, e_{i}\right\rangle\right|^{2}+\left|\left\langle e, f_{i}\right\rangle\right|^{2}}}
\end{aligned}
$$


suits our requirements.

1.5 Lemma. Let $\left(u_{i}: i \in I\right)$ be a net of unitary operators in a Hilbert space $H$. Let e, $f$ be orthogonal unit vectors in $H$ and $\left(\alpha_{i}\right),\left(\beta_{i}\right)$, nets in $\mathbb{C}$ such that $\left|\alpha_{i}\right|^{2}+\left|\beta_{i}\right|^{2}=1, i \in I$, and

$$
\alpha_{i} u_{i}(e)+\beta_{i} u_{i}(f) \rightarrow e_{0}, \quad-\vec{\beta}_{i} u_{i}(e)+\bar{\alpha}_{i} u_{i}(f) \rightarrow f_{0}
$$

for some orthonormal couple $e_{0}, f_{0} \in H$. Then for each orthonormal basis $x_{0}, y_{0}$ of $\mathbb{C} e_{0}+\mathbb{C} f_{0}$ there exists a pair $\left(\lambda_{i}\right),\left(\mu_{i}\right)$ of nets in $\mathbb{C}$ such that

$\left|\lambda_{i}\right|^{2}+\left|\mu_{i}\right|^{2}=1, u_{i}(e)=\lambda_{i} x_{0}+\mu_{i} y_{0}+o(1), u_{i}(f)=-\bar{\mu}_{i} x_{0}+\bar{\lambda}_{i} y_{0}+o(1)$

Proof. There exist $\gamma, \delta \in \mathbb{C}$ with

$$
x_{0}=\gamma e_{0}+\delta f_{0}, \quad y_{0}=-\bar{\delta} e_{0}+\bar{\gamma} f_{0}, \quad|\gamma|^{2}+|\delta|^{2}=1
$$

By assumption $\alpha_{i} u_{i}(e)+\beta_{i} u_{i}(f)=e_{0}+o(1),-\bar{\beta}_{i} u_{i}(e)+\bar{\alpha}_{i} u_{i}(f)=$ $f_{0}+o(1)$ whence

$$
\begin{aligned}
\left(\begin{array}{l}
u_{i}(e) \\
u_{i}(f)
\end{array}\right) & =\left(\begin{array}{cc}
\bar{\alpha}_{i} & -\beta_{i} \\
\bar{\beta}_{i} & \alpha_{i}
\end{array}\right)\left(\begin{array}{l}
e_{0} \\
f_{0}
\end{array}\right)+o(1) \\
& =\left(\begin{array}{cc}
\bar{\alpha}_{i} & -\beta_{i} \\
\bar{\beta}_{i} & \alpha_{i}
\end{array}\right)\left(\begin{array}{cc}
\bar{\gamma} & -\delta \\
\bar{\delta} & \gamma
\end{array}\right)\left(\begin{array}{l}
x_{0} \\
y_{0}
\end{array}\right)+o(1) \\
& =\left(\begin{array}{cc}
\lambda_{i} & \mu_{i} \\
-\bar{\mu}_{i} & \bar{\lambda}
\end{array}\right)\left(\begin{array}{l}
x_{0} \\
y_{0}
\end{array}\right)+o(1)
\end{aligned}
$$

where $\lambda_{i}:=\overline{\alpha_{i} \gamma}-\beta_{i} \bar{\delta}, \mu_{i}:=-\bar{\alpha}_{i} \delta-\beta_{i} \gamma$. Thus $\left|\lambda_{i}\right|^{2}+\left|\mu_{i}\right|^{2}=1, i \in I$, holds.

1.6 Lemma. Let $\left(u_{i}: i \in I\right)$ be a net of unitary operators in a Hilbert space $H$. Suppose that for every $e \in H$ there exists a net 
$\left(\alpha_{i}^{e}: i \in I\right)$ in $\mathbb{I}$ such that $\left(\alpha_{i}^{e} u_{i}(e)\right)$ converges in $H$. Then there exists a net $\left(\alpha_{i}: i \in I\right)$ such that the operators $\left(\alpha_{i} u_{i}\right)$ converge in the SOT of $\mathcal{L}(H)$.

Proof. Let us fix any unit vector $e \in H$ and write $e_{0}:=$ $\lim _{i} \alpha_{i}^{e} u_{i}(e)$. Consider any $f \in H$ lying orthogonally to $e$. It suffices to show that $\alpha_{i}^{e} u_{i}(f)$ is norm-convergent.

We may assume $\|f\|=1$. Set $f_{0}:=\lim _{i} \alpha_{i}^{f} u_{i}(f)$. Since unitary operators preserve the scalar product, $\alpha_{i}^{e} u_{i}(e) \perp \alpha_{i}^{f} u_{i}(f), i \in I$, and hence $e_{0} \perp f_{0}$. Moreover there is $g_{0} \in H$ such that

$$
\alpha_{i}^{e+f} u_{i}(e+f) \rightarrow g_{0}, \quad\left\|g_{0}\right\|=\|e+f\|=\sqrt{2} .
$$

We have

$$
\begin{aligned}
& u_{i}(e)=\overline{\alpha_{i}^{e}} e_{0}+o(1), u_{i}(f)=\overline{\alpha_{i}^{f}} f_{0}+o(1), \\
& u_{i}(e)+u_{i}(f)=u_{i}(e+f)=\overline{\alpha_{i}^{e+f}} g_{0}+o(1)
\end{aligned}
$$

Thus

$$
g_{0}=\alpha_{i}^{e+f} \overline{\alpha_{i}^{e}} e_{0}+\alpha_{i}^{e+f} \overline{\alpha_{i}^{f}} f_{0}+o(1) .
$$

Therefore there exist $\sigma, \tau \in \mathbb{T}$ with

$$
\alpha_{i}^{e+f} \overline{\alpha_{i}^{e}} \rightarrow \sigma, \alpha_{i}^{e+f} \overline{\alpha_{i}^{f}} \rightarrow \tau, g_{0}=\sigma e_{0}+\tau f_{0}
$$

It follows $\alpha_{i}^{f} / \alpha_{i}^{e} \rightarrow \sigma / \tau$ that is $\alpha_{i}^{e}=\tau \alpha_{i}^{f} / \sigma+o(1)$ and $\alpha_{i}^{e} u_{i}(f)=$ $\left(\frac{\tau}{\sigma} \alpha_{i}^{f}+o(1)\right) u_{i}(f)=\frac{\tau}{\sigma} \alpha_{i}^{f} u_{i}(f)+o(1) \rightarrow \frac{\tau}{\sigma} f_{0}$.

\section{NETS OF ISOMETRIES OF $\mathcal{C}(\Omega, E)$}

In this section $E$ denotes an arbitrary Cartan factor, $\Omega$ is a compact Hausdorff space and $\mathcal{U}:=\mathcal{C}(\Omega, E)$ is the $J B^{*}$-triple of continuous functions $f: \Omega \rightarrow E$ with the norm of the supremum. We recall that ([1] p. 142) a Banach space $X$ has the strong Banach-Stone property if whenever $M$ and $N$ are locally compact topological spaces and $l: \mathcal{C}_{0}(M, X) \rightarrow \mathcal{C}_{0}(N, X)$ is a surjective linear isometry, then $l$ can be represented in the form

$$
(l f)(\omega)=u(\omega) f[\tau(\omega)], \quad \omega \in N \quad f \in \mathcal{C}_{0}(M, X)
$$


for some homeomorphism $\tau: N \rightarrow M$ and some continuous function $u: \quad N \rightarrow \mathcal{U}(X)$, where $\mathcal{U}(X) \subset \mathcal{L}(X)$ is the group of surjective linear isometries of $X$ with the strong operator topology. If $l$ admits the representation (2), then we write $l:=(u, \tau)$

2.1 Lemma. In the above conditions, let $l_{1}=(u, \tau)$ and $l_{2}:=(v, \sigma)$ be two isometries of $\mathcal{U}$ with $\tau \neq \sigma$. Then there exists $f \in \mathcal{U}$ with $\|f\|=1$ such that ||$l_{1}(f)-l_{2}(f) \| \geq 1$.

Proof. Fix any $\omega_{0} \in \Omega$ with $\tau\left(\omega_{0}\right) \neq \sigma\left(\omega_{0}\right)$ and let $K \subset \Omega$ be a neighbourhood of $\omega_{0}$ such that $\tau(K) \cap \sigma(K)=\emptyset$. Take any $\varphi \in \mathcal{C}(\Omega)$ with values in $[0,1]$ such that

$$
\varphi_{\mid \gamma(K)} \equiv 1 \text { and } \varphi_{\mid \sigma(K)} \equiv 0
$$

Fix any $a \in E$ with $\|a\|=1$. Then $f:=\varphi \otimes a$ satisfies the requirements.

2.2 Lemma. Let $\mathcal{U}:=\mathcal{C}(\Omega, E)$ for an arbitrary Cartan factor $E$. Then $E$ has the strong Banach-Stone property, and for every $l \in \operatorname{Aut}(\mathcal{U})$ the representation (2) is unique.

Proof. By ([2], cor. 2.11) the centralizer $Z(E)$ of $E$ is a onedimensional space, hence by ([1], th. 8.11) $E$ has the strong BanachStone property.

The function $1 \otimes a$ is in $\mathcal{C}(\Omega, E)$ for every $a \in E$, hence $l(1 \otimes a)$ is well defined and

$$
l(1 \otimes a)(\omega)=u(\omega)[(1 \otimes a)(\tau(\omega))]=u(\omega) a
$$

which shows that $u$ is unique and is given by $u(\omega) a=l(1 \otimes a)(\omega)$ for all $\omega \in \Omega$. Now the uniqueness of (2) is an immediate consequence of (2.1).

2.3 Theorem. Let $\mathcal{U}:=\mathcal{C}(\Omega, E)$ for an arbitrary Cartan factor $E$ and let $\left(l_{i} ; i \in I\right)$ be a net in Aut $(\mathcal{U})$ such that for every $f \in \mathcal{U}, l_{i}(f)$ and $l_{i}^{-1}(f)$ converge in $U$. Then the mapping $l: f \mapsto \lim _{i} l_{i}(f)$ is surjective and for every $f \in \mathcal{U}$ we have $\lim _{i} l_{i}^{-1}(f)=l^{-1}(f)$ in $\mathcal{U}$. 
Proof. We have

$$
l_{i}(f)(\omega)=u_{i}(\omega) f\left[\tau_{i}(\omega)\right] \rightarrow l(f)(\omega), \quad(\omega \in \Omega, f \in \mathcal{U})
$$

By (2.1) this implies $\tau_{i}=\tau$ for some homeomorphism of $\Omega$ and all $i \geq i_{0}$. Applying (3) to $f:=1 \otimes a$ for $a \in E$, we get

$$
l_{i}(1 \otimes a)(\omega)=u_{i}(\omega) a \rightarrow l(1 \otimes a)(\omega)
$$

uniformly for $\omega \in \Omega$. Hence to every $\omega \in \Omega$ and every $a \in E$, the net $\left(u_{i}(\omega) a, i \in I\right)$ converges in $E$. Thus $u(\omega): a \mapsto \lim _{i} u_{i}(\omega) a$ is in an isometry of $E$ and $u \in \mathcal{C}(\Omega, \mathcal{L}(E))$. We claim that $u(\omega)$ is surjective for every $\omega \in \Omega$. Indeed, by (2.1) and (2.2) we have

$$
\left(l_{i}^{-1} f\right)(\omega)=v_{i}(\omega) f\left[\sigma_{i}(\omega)\right]
$$

where $v_{i}(\omega)=u_{i}(\omega)^{-1}, \sigma_{i}=\tau_{i}^{-1}$ for all $\omega \in \Omega, i \in I$, and $\sigma_{i}=\sigma$ for some homeomorphism $\sigma: \Omega \rightarrow \Omega$ and $i \geq i_{0}$. Reasoning as we did before, $\left(v_{i}(\omega)\right)$ tends to $v(\omega)$ for some continuous function $v: \Omega \rightarrow$ $\mathcal{L}(E)$. Moreover, we have

$$
u(\omega) \circ v(\omega)=i d_{E}=v(\omega) \circ u(\omega), \quad(\omega \in \Omega)
$$

since otherwise we would have

$$
u\left(\omega_{0}\right) v\left(\omega_{0}\right) a=b \neq a
$$

for some $\omega_{0} \in \Omega$ and $a \in E$. But then

$$
\begin{aligned}
\|a-b\| & =\left\|v_{i}\left(\omega_{0}\right) u_{i}\left(\omega_{0}\right) a-v\left(\omega_{0}\right) u\left(\omega_{0}\right) a\right\| \\
& \leq\left\|v_{i}\left(\omega_{0}\right)\left[u_{i}\left(\omega_{0}\right) a-u\left(\omega_{0}\right) a\right]\right\|+\left\|\left[v_{i}\left(\omega_{0}\right)-v\left(\omega_{0}\right)\right] u\left(\omega_{0}\right) a\right\| \\
& \leq\left\|u_{i}\left(\omega_{0}\right) a-u\left(\omega_{0}\right) a\right\|+\left\|\left[v_{i}\left(\omega_{0}\right)-v\left(\omega_{0}\right)\right] u\left(\omega_{0}\right) a\right\| \rightarrow 0
\end{aligned}
$$


which contradicts $a \neq b$. Thus $u$ is surjective and so $u \in \operatorname{Aut}(E)$. Clearly

$$
l(g)(\omega):=\lim _{i} l_{i} g(\omega)=u(\omega) g[\tau(\omega)]
$$

holds for all $\omega \in \Omega$ and $g \in \mathcal{U}$, i.e., we have $l=(u, \tau)$ which shows that $l$ is surjective.

\section{NETS OF ISOMETRIES OF SPECIAL CARTAN FAC- TORS}

If $\Omega$ is a single point and $E$ is special, then the above results can be improved.

3.1 Theorem. Let $\mathcal{U}$ be a special Cartan factor and let $\left(l_{i} ; i \in I\right)$ be a net in Aut $(\mathcal{U})$ such that $\left(l_{i}(a)\right)$ and $\left(l_{i}^{-1}(a)\right)$ converge in $\mathcal{U}$ for every $a \in$ at $(\mathcal{U})$. Then there are a subnet $\left(i_{k}: k \in \mathcal{K}\right)$ and an $l \in \operatorname{Aut}(\mathcal{U})$ such that $l_{i_{k}}(x) \rightarrow l(x)$ holds in the SOT for every $x \in \mathcal{U}$.

Proof. We make a type by type discussion. Suppose $k=1$. By ([10], Satz 4) every $l_{i}$ admits one of these two representations:
(a) $l_{i}(x)=u_{i} \circ x \circ v_{i}$
(b) $l_{i}(x)=u_{i} \circ x^{t} \circ v_{i} \quad(x \in \mathcal{U})$

for some $u_{i} \in \mathcal{U}(K), v_{i} \in \mathcal{U}(H)$ or, respectively, for some surjective isometries $u_{i}, v_{i}: H \rightarrow K$. We claim that for large enough indices (say $i \geq i_{0}$ ) either all $l_{i}$ have the form (a) or all $l_{i}$ have the form (b). Indeed, fix a non zero atom $a \in$ at $(\mathcal{U})$; then $\left(l_{i}(a)\right)$ is a Cauchy net in $\mathcal{U}$ and the claim follows easily. Clearly it suffices to consider the possibility (a) since (b) is quite similar. Fix two unit vectors $e \in K$ and $f \in H$ arbitrarily. Then the operator $e \otimes f^{*}$ is an atom of $\mathcal{U}$ and

$$
u_{i} \circ\left(e \otimes f^{*}\right) \circ v_{i}=u_{i}(e) \otimes v_{i}^{*}(f)^{*} .
$$

By (1.2) there exists a net $\left(\alpha_{i}: i \in I\right)$ in $\mathbb{T}$ such that $\left(\alpha_{i} u_{i}(e)\right)$ and $\left(\alpha_{i} v_{i}^{*}(f)\right)$ converge in $K$ and $H$ respectively. We claim that $\left(\alpha_{i}\right)$ does not depend on the pair $e, f$. Indeed, consider any couple of unit vectors $g \in K, h \in H$. By assumption, the operators

$$
u_{i} \circ\left(g \otimes f^{*}\right) \circ v_{i}^{*}=u_{i}(g) \otimes v_{i}^{*}(f)^{*}
$$


converge in $\mathcal{U}$. Hence we have

$$
\begin{aligned}
& \left\|\alpha_{i} u_{i}(g)-\alpha_{j} u_{j}(g)\right\| \\
& =\left\|\left[\alpha_{i} u_{i}(g)\right] \otimes\left[\alpha_{i} v_{i}^{*}(f)\right]^{*}-\left[\alpha_{j} u_{j}(g)\right] \otimes\left[\alpha_{i} v_{i}^{*}(f)\right]^{*}\right\| \\
& \leq \|\left[\alpha_{i} u_{i}(g)\right] \otimes\left[\alpha_{i} v_{i}^{*}(f)\right]^{*}-\left[\alpha_{j} u_{j}(g)\right] \otimes\left[\alpha_{j} v_{j}^{*}(f)\right]^{*} \\
& +\left[\alpha_{j} u_{j}(g)\right] \otimes\left[\alpha_{j} v_{j}^{*}(f)-\alpha_{i} v_{i}^{*}(f)\right]^{*} \| \\
& \leq\left\|\left[\alpha_{i} u_{i}(g)\right] \otimes\left[\alpha_{i} v_{i}^{*}(f)\right]^{*}-\left[\alpha_{j} u_{j}(g)\right] \otimes\left[\alpha_{j} v_{j}^{*}(f)\right]^{*}\right\| \\
& +\left\|\alpha_{j} v_{j}^{*}(f)-\alpha_{i} v_{i}^{*}(f)\right\| \rightarrow 0
\end{aligned}
$$

Thus $\left(\alpha_{i} u_{i}(g)\right)$ is a Cauchy net in $K$. To deduce the convergence of $\left(\alpha_{i} v_{i}^{*}(h)\right)$, we consider the adjoint space $\mathcal{L}(K, H)$ and apply the same argument to the convergent net of operators

$$
\left[\alpha_{i} v_{i}^{*}(h)\right] \otimes\left[\alpha_{i} u_{i}(g)\right]^{*}=\left[u_{i}(g) \otimes v_{i}^{*}(h)^{*}\right]^{*}
$$

Therefore

$$
\alpha_{i} u_{i} \rightarrow u \quad \text { and } \quad \alpha_{i} v_{i}^{*} \rightarrow v^{*}
$$

in the SOT of $\mathcal{U}$ for some partial isometries $u \in \mathcal{L}(K), v^{*} \in \mathcal{L}(H)$. But $\left(l_{i}^{-1}(a)\right)$ also converges in $\mathcal{U}$ for every atom $a \in$ at $(\mathcal{U})$. Since

$$
l_{i}^{-1}(x)=v_{i}^{*} \circ x \circ v_{i}^{*}, \quad(x \in \mathcal{U}, i \in I),
$$

by the same argument there exists a net $\left(\beta_{i}\right)$ in $\mathbb{I}$ such that $\beta_{i} u_{i}^{*} \rightarrow r^{*}$ and $\beta_{i} v_{i} \rightarrow s$ in the SOT of $\mathcal{U}$ for some partial isometries $r^{*} \in \mathcal{L}(K), s \in$ $\mathcal{L}(H)$. By setting $\gamma_{i}:=\alpha_{i} \beta_{i}, i \in I$, we have $\gamma_{i} 1_{H}=\gamma_{i} u_{i} \circ u_{i}^{*} \rightarrow u \circ r^{*}$. By compactness of $\mathbb{T}$, there is a subnet $\left(\gamma_{i_{k}}\right)$ and some $\gamma_{0} \in \mathbb{T}$ such that $\gamma_{i_{k}} \rightarrow \gamma_{0}$ and so $\gamma_{i_{k}} 1_{H} \rightarrow \gamma_{0} 1_{H}$. Therefore, $\gamma_{0} 1_{H}=u$ o $r^{*}$ and $u, r^{*}$ are surjective. Similarly $v^{*}, s$ are surjective. By ([12] Remark 4.10, p. 84 ), on the unitary group $\mathcal{U}(H)$ the SOT coincides with the SSOT. As 
the adjoint operation $x \mapsto x^{*}$ is SSOT continuous, (4) yields $\bar{\alpha}_{i} v_{i} \rightarrow v$. Multiplication in $\mathcal{U}$ restricted to bounded sets is jointly continuous with respect to the SOT, hence

$$
l_{i}(x)=\left(\alpha_{i} u_{i}\right) \circ x \circ\left(\bar{\alpha}_{i} v_{i}\right) \rightarrow l(x):=u \circ x \circ v
$$

in the SOT and $l \in \operatorname{Aut}(\mathcal{U})$.

Suppose $k=2$. By [5] we have

$$
l_{i}(x)=u_{i} \circ x \circ u_{i}^{t}, \quad(x \in \mathcal{U}, i \in I)
$$

for some unitary operators $u_{i} \in \mathcal{U}(H)$. Fix any atom $e \otimes \bar{e}^{*}$ in $\mathcal{U}$. By assumption, the operators

$$
u_{i} \circ\left(e \otimes \bar{e}^{*}\right) \circ u_{i}^{t}=u_{i}(e) \otimes \overline{u_{i}(e)^{*}}
$$

converge in $\mathcal{U}$. By (1.3) applied to $e_{i}:=u_{i}(e)$, for any unit vector $e \in H$ there exists a net $\left(\varepsilon_{\dot{i}}^{e}: i \in I\right)$ in $\{-1,1\}$ such that $\varepsilon_{i}^{e} u_{i}(e)$ converge. Fix a unit vector $e \in H$ arbitrarily. We claim that $\varepsilon_{i}^{e} u_{i}(f)$ converges in $H$ whenever $f$ is a unit vector lying orthogonally to $e$. Write $e_{0}:=\lim _{i} \varepsilon_{i}^{e} u_{i}(e), f_{0}:=\lim _{i} \varepsilon_{i}^{f} u_{i}(f), g_{0}:=\lim _{i} \varepsilon_{i}^{g} u_{i}(g)$ where $g:=\frac{1}{\sqrt{2}}(e+f)$. Thus

$$
\varepsilon_{i}^{x} u_{i}(x)=x_{0}+o(1)
$$

for $x=e, f, g$ and $x_{0}=e_{0}, f_{0}, g_{0}$ respectively. Since $\varepsilon_{i}^{x} \in\{-1,1\}$ and so $\left(\varepsilon_{i}^{x}\right)^{2}=1$, we have

$$
u_{i}(x)=\varepsilon_{i}^{x} x_{0}+o(1)
$$

whence

$$
\varepsilon_{i}^{e} e_{0}+\varepsilon_{i}^{f} f_{0}=u_{i}(e)+u_{i}(f)+o(1)=\sqrt{2} u_{i}(g)+o(1)=\sqrt{2} \varepsilon_{i}^{g} g_{0}+o(1)
$$

Since $e_{0} \perp f_{0}$, it follows

$$
\varepsilon_{i}^{\mathrm{e}}=\sqrt{2}\left\langle g_{0}, e_{0}\right\rangle \varepsilon_{i}^{g}+o(1), \quad \varepsilon_{i}^{f}=\sqrt{2}\left\langle g_{0}, f_{0}\right\rangle \varepsilon_{i}^{g}+o(1) .
$$


Hence $\varepsilon_{i}^{e} \varepsilon_{i}^{f}=$ constant $+o(1)$. Therefore $\varepsilon_{i}^{e} u_{i}(e)$ and $\varepsilon_{i}^{e} u_{i}(f)$ converge simultaneously, and we can define a net $\left(\varepsilon_{i}, i \in I\right)$ not depending on $e \in H$ so that $\varepsilon_{i} u_{i} \rightarrow u$ in the SOT for some partial isometry $u \in \mathcal{L}(H)$. The same argument applied to

$$
l_{i}^{-1}(x)=u_{i}^{*} \circ x \circ u_{i}^{* t} \quad(x \in \mathcal{U}, i \in I)
$$

yields the existence of a net $\left(\eta_{i}, i \in I\right)$ in $\{1,-1\}$ such that $\eta_{i} u_{i}^{*} \rightarrow v^{*}$ in the SOT for some partial isometry $v^{*} \in \mathcal{L}(H)$. But then

$$
\varepsilon_{i} \eta_{i} 1_{H}=\varepsilon_{i} \eta_{i} u_{i} \circ u_{i}^{*} \rightarrow u \circ v^{*}
$$

in the SOT. By taking a subnet $\left(i_{k}: k \in \mathcal{K}\right)$ we may assume that $\rho_{i_{k}}:=\varepsilon_{i_{k}} \eta_{i_{k}} \rightarrow \rho_{0}$ for some $\rho_{0} \in \mathbb{T}$, hence $\rho_{i_{k}} 1_{H} \rightarrow \rho_{0} 1_{H}$ and so $\rho_{0} 1_{H}=u \circ v^{*}$ which shows that $u, v^{*} \in \mathcal{U}(H)$. Thus $l(x):=u \circ x \circ u^{t}$ defines an $l \in \operatorname{Aut}(\mathcal{U})$ and $l_{i}(x) \rightarrow l(x)$ in the SOT for every $x \in \mathcal{U}$.

Suppose $k=3$. By [5], we have

$$
l_{i}(x)=u_{i} \circ x \circ u_{i}^{t}, \quad(x \in \mathcal{U}, i \in I)
$$

for some $u_{i} \in \mathcal{U}(H)$. Fix any atom $e \otimes \bar{f}^{*}-f \otimes \bar{e}^{*}$ in $\mathcal{U}$. By assumption, the operators

$$
u_{i} \circ\left(e \otimes \bar{f}^{*}-f \otimes \bar{e}^{*}\right) \circ u_{i}^{t}=u_{i}(e) \otimes \overline{u_{i}(f)^{*}}-u_{i}(f) \otimes \overline{u_{i}(e)^{*}}
$$

converge in $\mathcal{U}$, whence by $(1.4)$ there are nets $\left(\alpha_{i}\right)$ and $\left(\beta_{i}\right)$ in $\bar{\Delta}$ such that

$$
\alpha_{i} u_{i}(e)+\beta_{i} u_{i}(f) \rightarrow e_{0} \quad \text { and } \quad-\bar{\beta}_{i} u_{i}(f)+\bar{\alpha}_{i} u_{i}(e) \rightarrow f_{0}
$$

hold in $H$ for some orthogonal unit vectors $e_{0}, f_{0} \in H$. By (1.5) if we fix a basis $x_{0}, y_{0}$ in $\mathbb{C} e_{0}+\mathbb{C} f_{0}$, then there are nets $\left(\lambda_{i}\right),\left(\mu_{i}\right)$, in $\bar{\Delta}$ such that

$$
u_{i}(e)=\lambda_{i} x_{0}+\mu_{i} y_{0}+o(1) \quad \text { and } \quad u_{i}(f)=-\bar{\mu}_{i} x_{0}+\bar{\lambda}_{i} y_{0}+o(1)
$$


By compactness of $\bar{\Delta}$, there is a subnet $\left(i_{k} ; k \in \mathcal{K}\right)$ such that $\lambda_{i_{k}} \rightarrow \lambda_{0}$, and $\mu_{i_{k}} \rightarrow \mu_{0}$. From (5) we get in particular that $\left(u_{i_{k}}(e)\right)$ converges in $H$

$$
u_{i_{k}}(e) \rightarrow \lambda_{0} x_{0}+\mu_{0} y_{0}
$$

By (1.6) the subnet $\left(i_{k} ; k \in \mathcal{K}\right)$ does not depend on the pair of vectors $e, f$. Thus $\left(u_{i_{k}}: k \in \mathcal{K}\right)$ is well defined and $u_{i_{k}} \rightarrow u$ in the SOT for some partial isometry $u \in \mathcal{L}(H)$. A similar argument applied to $\left(l_{i_{k}}^{-1}\right)$ gives the existence of a subnet (still denoted by $\left(i_{k}: k \in \mathcal{K}\right)$ ) such that $u_{i_{k}}^{*} \rightarrow v$ in the SOT for some partial isometry $v \in \mathcal{L}(H)$ and a standard reasoning gives that $u \in \mathcal{U}(H)$. Therefore $l(x):=u \circ x \circ u^{t}$ for $x \in U$ satisfies the requierements. Notice that, contrary to the other types of Cartan factors, the consideration of a subnet of $\left(l_{i}\right)$ has now been necesary.

Suppose $k=4$. Let $\mathcal{H}$ be the Hilbert space associated to $\mathcal{U}$. Thus non zero atoms of $\mathcal{U}$ are the same as unit vectors of $\mathcal{H}$. By [6] we have

$$
l_{i}(x)=\lambda_{i} u_{i}(x), \quad(i \in I, x \in \mathcal{U})
$$

for some $\lambda_{i} \in \mathbb{T}$ and some unitary operators $u_{i} \in \mathcal{U}(\mathcal{H})$ such that $u_{i}(x)^{*}=u_{i}\left(x^{*}\right)$ for $x \in \mathcal{U}$. Suppose that, for every $x \in \mathcal{H}$, the net

$$
l_{i}(x)=\lambda_{i} u_{i}(x), \quad(i \in I)
$$

converges in $\mathcal{U}$, (hence also in $\mathcal{H}$ ). By taking a subnet we may assume that $\lambda_{i} \rightarrow \lambda_{0}$ for some $\lambda_{0} \in \mathbb{T}$. By $(7),\left(u_{i}\right)$ converges pointwise to a partial isometry $u$ of $\mathcal{H}$. A similar argument with $\left(l_{i}^{-1}\right)$ yields $u_{i}^{*} \rightarrow v^{*}$ pointwise on $\mathcal{H}$ and one easily sees that $u \circ v^{*}=1_{H}=v^{*} \circ u$. Thus $u \in \mathcal{U}(\mathcal{H})$. Since the star operation is norm continuous in $\mathcal{U}$, we have

$$
[u(x)]^{*}=\left[\lim _{i \rightarrow \infty} u_{i}(x)\right]^{*}=\lim _{i \rightarrow \infty}\left[u_{i}(x)^{*}\right]=\lim _{i \rightarrow \infty}\left[u_{i}^{*}(x)\right]=u^{*}(x)
$$

Then $l(x):=\lambda_{0} u(x)$ for $x \in \mathcal{U}$ satisfies the requirements. 


\section{NETS OF HOLOMORPHIC AUTOMORPHISMS IN CARTAN FACTORS}

If $\mathcal{U}$ is an arbitrary Cartan factor with open unit ball $D$ and $g \in$ $G:=\operatorname{Aut}(D)$, then by [9] we have $g=g_{a} \circ \lambda=\lambda \circ g_{\lambda^{-1}(a)}$ for some $\lambda \in \operatorname{Aut}(\mathcal{U})$ and $g_{a} \in G$ with $a:=g(0)=g_{a}(0)$ and $g_{a}^{-1}=g_{-a}$. By [7] every $g \in G$ extends to a uniformly continuous holomorphic function a neighbourhood of $\bar{D}$ and $g(\partial D) \subset \partial D$.

4.1 Theorem. Let $\mathcal{U}$ be a special Cartan factor and $\left(h_{i}: i \in I\right)$ a net in $G$ such that:

(i) For every $x \in D$, the net $\left(h_{i}(x)\right)$ is norm convergent to a limit $h(x) \in \mathcal{U}$.

(ii) $\left(h_{i}(0)\right)$ is bounded away from $\partial D$.

(iii) $\left(h_{i}^{-1}(e)\right)$ is norm convergent for every $e \in$ at $(\mathcal{U})$.

Then $h$ belongs to $G$, and if the set $I$ is countable, then $\left(h_{i}\right)$ converges to $h$ in $\mathcal{U}$ uniformly on every compact subset of $D$.

Proof. Let $h_{i}$ have the representation $h_{i}=g_{a_{i}} \circ \lambda_{i}$ where $a_{i}:=h_{i}(0)$ for $i \in I$. Notice that $0 \in \operatorname{at}(\mathcal{U})$. By (i) and (ii) we have $a_{i} \rightarrow a \in D$ in the norm topology of $\mathcal{U}$. Thus by [8], $g_{a_{i}}$ tends to $g_{a}$ uniformly on a neighbourhood of $\bar{D}$. By the uniform continuity of $g_{a}$ on $\bar{D}$ we have for every $x \in D$

$$
\begin{aligned}
\left\|\lambda_{i}(x)-\lambda_{j}(x)\right\| & =\left\|g_{-a_{i}} h_{i}(x)-g_{-a_{i}} h_{j}(x)\right\| \\
& \leq\left\|g_{-a_{i}} h_{i}(x)-g_{-a_{i}} h_{i}(x)\right\|+\left\|g_{-a} h_{i}(x)-g_{-a} h_{j}(x)\right\| \\
& +\left\|g_{-a} h_{j}(x)-g_{-a_{j}} h_{j}(x)\right\| \rightarrow 0
\end{aligned}
$$

which shows that $\left(\lambda_{i}(x)\right)$ converges in $\mathcal{U}$ for every $x \in D$, hence also for every $y \in \mathcal{U}$ (and in particular for every atom $e \in \operatorname{at}(\mathcal{U})$ ) by the linearity of the $\lambda_{i}$.

We now consider the net $\left(h_{i}^{-1}: i \in I\right)$, where $h_{i}^{-1}=\lambda_{i}^{-1} \circ g_{-a_{i}}=$ $g_{b_{i}} \circ \mu_{i}$ with $\mu_{i}:=\lambda_{i}^{-1}$ and $b_{i}:=-\mu_{i}\left(a_{i}\right)$ for $i \in I$. Then $\left(b_{i}\right)$ is bounded away from $\partial D$ because so is $\left(a_{i}\right)$ and $\left\|\mu_{i}\right\|=1$, hence by (iii) $b_{i} \rightarrow b$ in $\mathcal{U}$ 
for some $b \in D$. Thus $g_{b_{i}}$ tends to $g_{b}$ uniformly on a neighbourhood of $\bar{D}$. If in the above inequalities we replace $\lambda_{i}, g_{-a_{i}}, h_{i}$ and $x$ by $\mu_{i}, g_{b_{i}}, h_{i}^{-1}$ and $e$ respectively and use (iii), then we get that $\left(\mu_{i}(e)\right)$ is a Cauchy net in $\mathcal{U}$ for every atom $e \in \operatorname{at}(\mathcal{U})$.

Therefore (3.1) applies to our case, and there exists a subnet $\left(i_{k}\right.$ : $k \in \mathcal{K})$ and a $\lambda \in \operatorname{Aut}(\mathcal{U})$ such that $\lambda_{i_{k}}(x) \rightarrow \lambda(x)$ in the SOT for every $x \in \mathcal{U}$. However, as seen before, the whole net $\left(\lambda_{i}(x)\right)$ converges in $\mathcal{U}$; therefore we must have $\lambda_{i_{k}}(x) \rightarrow \lambda(x)$ in the norm of $\mathcal{U}$. Then $g_{a} \circ \lambda$ lies in $G$ because $\lambda$ is surjective, and $h_{i}(x) \rightarrow g_{a} \circ \lambda(x)$ in $\mathcal{U}$ for every $x \in D$, hence $h=g_{a} \circ \lambda$. The remainder of the proof is an easy consequence of the Banach-Steinhauss theorem.

\section{References}

[1] Behrends, E.: $M$-structure and the Banach-Stone theorem. Lecture Notes in Mathematics Vol. 736. Berlin-Heidelberg-New York: Springer 1979.

[2] Dineen, S. and Timony, R.M.: The centroid of a $J B^{*}$-triple system. Math. Scand. 62, (1988), 327-342.

[3] Harris, L.A.: Bounded symmetric homogeneous domains in infinite dimensional spaces. Lecture Notes in Mathematics Vol. 364. BerlinHeidelberg-New York: Springer 1973.

[4] Harris, L.A.: Analytic invariants and the Schwarz-Pick inequality. Israel J. Maths. 34, (1979), 177-197.

[5] Hervés, J.: Linear isometries of Cartan factors in infinite dimensions. Ann. di Mat. Pura ed Appl. (IV) vol. CXLII, (1985), 371-379.

[6] Hervés, J. and Isidro, J.M.: Isometries and automorphisms of the spaces of spinors. Rev. Mat. Univ. Compl. Madrid 5, (1992), 193-200.

[7] Isidro, J.M. and Kaup, W.: Weak continuity of holomorphic automorphisms in $J B^{*}$-triples. Math. Z. 210, (1992), 277-288.

[8] Isidro, J.M. and Kaup, W.: Determining boundary sets of bounded symmetric domains. Manuscripta Math. 81 (1993) 149-159.

[9] Kaup, W.: A Riemann mapping theorem for bounded symmetric domains in complex Banach spaces. Math. Z. 183, (1983), 503-529. 
[10] Kaup, W.: Über die Automorphismen Graßmannscher Mannigfaltigkeiten unendlicher Dimension. Math. Z. 144, (1975), 75-96.

[11] Loos, O.: Bounded symmetric domains and Jordan pairs. Mathematical Lectures, University of California at Irvine 1977.

[12] Takesaki, MP.: Theory of operator algebras I. Springer-Verlag New York 1979.

Departamento de And́lisis Matemático

Facultad de Matemáticas

15706 Santiago de Compostela

Spain

jmisidro@zmat.usc.es
Bolyai Intézete

Aradi Vértanuk tere 1

6720 Szeged

Hungary

Recibido: 14 de Julio de 1993

Revisado: 3 de Mayo de 1994 